\title{
A new mixed micellar preparation for oral vitamin K prophylaxis: randomised controlled comparison with an intramuscular formulation in breast fed infants
}

Frank R Greer, Sharon P Marshall, Rebecca R Severson, David A Smith, Martin J Shearer, Daniel G Pace, Pieter H Joubert

\begin{abstract}
Objective-To compare a new oral preparation of vitamin $\mathrm{K}_{1}$ (Konakion $M M$ ) containing lecithin and glycocholic acid with a standard intramuscular (IM) preparation during the first 8 weeks of life in exclusively breast fed infants.
\end{abstract}

Methods-Infants were randomised at birth to the IM group (1 mg vitamin $\mathrm{K}$ ) or the oral group ( $2 \mathrm{mg}$ given at birth and repeated at 7 and 30 days of life). Prothrombin time (INR), plasma vitamin $K_{1}$, and PIVKA II (undercarboxylated prothrombin) were monitored at 14,30 , and 56 days of age.

Results-Seventy nine infants were randomised to the oral group and 77 to the IM group. Sixty seven infants in each group completed eight weeks of the study. Prothrombin times did not differ between the two groups. Mean (SD) plasma vitamin $\mathrm{K}_{1}$ values (in $\mathrm{ng} / \mathrm{ml}$ ) decreased in both groups over time, but were higher in the oral group at 14 and 56 days: 2.0 (1.6) $v 1.3$ (1.1) at 14 days; $0.5(0.3) v 0.5(0.7)$ at 30 days; and $0.5(0.8) v 0.2(0.2)$ at 56 days of life. PIVKA II was raised ( $\geqslant 0.1 \mathrm{AU} / \mathrm{ml})$ in cord blood in $47 \%$ of the infants. By 14 days, only one infant in each group had a raised PIVKA II value and both of these initially had high concentrations of PIVKA II in cord blood. At 30 days, there were no raised PIVKA II values. At 56 days, there were no raised PIVKA II values in the oral group, although three infants in the IM group had raised values.

Conclusions-Plasma vitamin $\mathrm{K}$ concentrations were at least equal or significantly higher in babies given oral vitamin $K$ supplements compared with IM treated babies at the time points measured. Through the first 8 weeks of life, multiple doses of the new oral preparation maintain haemostasis and vitamin $K$ status in breast fed infants at least equal to that of the intramuscular preparation.

(Arch Dis Child 1998;79:300-305)

Keywords: vitamin $\mathrm{K}_{1}$ deficiency; breast feeding; haemorrhagic disease
Vitamin $\mathrm{K}_{1}$ deficiency can cause a bleeding disorder in infants through the first 3 months of life. It is mainly a problem in breast fed infants, particularly those who have disorders of intestinal absorption (biliary atresia, $\alpha-1$ antitrypsin deficiency, etc). ${ }^{12}$ The efficacy of newborn intramuscular vitamin $\mathrm{K}$ prophylaxis for prevention of this bleeding problem has been well established. However, in some Western countries where oral prophylaxis of vitamin $\mathrm{K}$ has been promoted over the traditional intramuscular route, the problem of haemorrhagic disease of the newborn has become a significant concern once again..$^{3-8}$ This probably reflects the variable intestinal absorption of presently available preparations. ${ }^{9}$

There is a new oral formulation of vitamin $\mathrm{K}$ available for prophylactic use in newborns. This new preparation, Konakion $M M$, is prepared with the phospholipid lecithin and the bile salt glycocholic acid, because vitamin $\mathrm{K}$ requires emulsification and the presence of bile salts for its absorption. Limited studies have shown that this "mixed micelle" solution has greater reliability of absorption in both normal newborns and children with chronic cholestasis. $^{1011}$ However, it has not been compared directly with the intramuscular form in newborn infants except in one small study during the first few weeks of life, in which infants received a single dose of Konakion MM. ${ }^{11}$ The purpose of our study was to make this comparison in breast feeding infants. We hypothesised that multiple doses of Konakion MM given during the 1 st month of life to exclusively breast feeding infants would be well tolerated, and that their vitamin $K_{1}$ status would not differ from that of breast feeding infants who received a single intramuscular dose of vitamin $\mathrm{K}_{1}$ at birth.

\section{Methods}

Our study was carried out at two private hospitals in southern Wisconsin: Meriter Hospital in Madison and Franciscan-Skemp Medical Center in La Crosse, where most of the infants are breast feeding at the time of discharge. All mothers participating in the study were recruited before delivery. At both hospitals, a 
single $1 \mathrm{mg}$ injection of vitamin $\mathrm{K}_{1}$ is given routinely after birth to all infants. Inclusion criteria included a gestational age of $\geqslant 37$ weeks or $\leqslant 42$ weeks, a birth weight $>2500 \mathrm{~g}$, singleton, mother planning to breast feed her infant until at least 2 months of age, absence of major congenital or malabsorptive disorders, no maternal history of anticonvulsant treatment during pregnancy, and an Apgar score of $\geqslant 7$ at five minutes. Written informed consent was obtained from all mothers and the protocols were approved by the human subject committees at both hospitals.

Immediately after birth, infants were randomised to receive either a standard $1 \mathrm{mg}$ intramuscular injection of vitamin $\mathrm{K}_{1}$ (Konakion) or a $2 \mathrm{mg}$ oral dose of the experimental preparation (Konakion MM). Randomisation was accomplished using a computer generated list, randomised in blocks of four. Because of the different methods of drug administration, subjects and clinical study personnel were not blinded to the different treatments. Both drugs were supplied in single dose glass vials by Hoffmann-La Roche. The intramuscular vitamin $\mathrm{K}$ was given according to standard hospital practice. The oral preparation was given by drawing up $0.2 \mathrm{ml}(2 \mathrm{mg})$ of the medication in a $1 \mathrm{ml}$ tuberculin syringe, and dispensing it on to the back of the infant's tongue. The infant was observed for five minutes and if any spitting was noted, the infant was given a dose of intramuscular vitamin $\mathrm{K}$ and dropped from the study. The oral dose was repeated during home visits by registered nurses, at 7 and 30 days of age, to give a total dose of $6 \mathrm{mg}$ in the 1 st month of life. If an infant in the oral group dropped out before completing the eight week study, an intramuscular dose of vitamin $\mathrm{K}_{1}$ was given .

All mothers were expected to breast feed their infants exclusively. Solid foods were not introduced. Mothers were supplied with a limited quantity of Similac PM 60/40 without added vitamin $\mathrm{K}_{1}$ (supplied by Ross Laboratories, Columbus, Ohio, USA) for emergency use only. If more than one bottle a week of the formula was used, infants were withdrawn from the study. Potentially adverse events pertaining to all major organ systems were monitored.

BLOOD SAMPLING AND LABORATORY METHODS Cord blood samples $(5 \mathrm{ml})$ were collected in EDTA coated glass tubes from all placentas. Additional venous or capillary (heel prick) blood samples $(3-4 \mathrm{ml})$ were collected at 2,4 , and 8 weeks after birth into glass tubes containing EDTA. A topical anaesthetic cream (EMLA) was used for blood sampling. Plasma vitamin $\mathrm{K}_{1}$ concentrations and prothrombin time were measured at 14, 30, and 56 days after birth. Vitamin $\mathrm{K}_{1}$ was not measured in cord blood, where it is generally undetectable with the assay used in our study. PIVKA II (protein induced in the absence of vitamin $K_{1}$ or des-gamma-carboxy-prothrombin) was measured in all blood samples. Plasma for vitamin $K_{1}$ and PIVKA II was separated by centrifugation from red cells and stored at $-20^{\circ} \mathrm{C}$ until analysis. Laboratory personnel were blinded to the infant's randomisation.

Vitamin $\mathrm{K}_{1}$ was assayed in plasma by a modification $^{12}$ of an earlier multistage procedure. ${ }^{13}$ After extraction of $0.5 \mathrm{ml}$ of plasma with hexane, the lipid extract was purified by normal phase, high performance liquid chromatography (HPLC), and analysed with reversed phase HPLC, using electrochemical detection in the redox mode. Menaquinone- 6 was used as the internal standard. The lower limit of detection was $0.05 \mathrm{ng} / \mathrm{ml}$. The intraassay precision for three plasma pools at mean measured concentrations of $0.30,0.76$, and $3.66 \mathrm{ng} / \mathrm{ml}$ was $5.0 \%, 7.2 \%$, and $3.3 \%$, respectively. The interassay precision over the period of our study was $11.3 \%, 10.2 \%$, and $9.8 \%$, respectively, for the same plasma pools. The normal ranges in adults are: fasting, 0.17$0.68 \mathrm{ng} / \mathrm{ml}$ (mean, 0.41) and non-fasting, $0.15-1.55 \mathrm{ng} / \mathrm{ml}$ (mean, 0.66), with a "low value" being $<0.15 \mathrm{ng} / \mathrm{ml}$.

PIVKA II was determined by an enzyme linked immunosorbent assay (ELISA) using a commercial kit (Eitest mono P-II; Eisai Co Ltd, Tokyo, Japan) with monoclonal antibodies specific for the inactive des-gamma-carboxy form of prothrombin. ${ }^{14}$ Results using this assay are expressed as AU/ml (arbitrary units $/ \mathrm{ml}$ ), with one AU corresponding to $1 \mu \mathrm{g}$ of purified prothrombin. The maximum concentration measured in a group of normal, healthy adults was $<0.1 \mathrm{AU} / \mathrm{ml}$. Therefore, this value was taken as the cut off point (or upper limit of normal) of the assay and a value $\geqslant 0.1 \mathrm{AU} / \mathrm{ml}$ was reported as PIVKA II antigen positive. The calibration plots of the log of optical density $v$ PIVKA II concentration were linear over the range of standards ranging between 0.0625 and $2.0 \mathrm{AU} / \mathrm{ml}$. For our study, three quality control plasma pools corresponding to low $(\sim 0.1 \mathrm{AU} / \mathrm{ml})$, middle $(\sim 0.5 \mathrm{AU} / \mathrm{ml})$, and high $(\sim 1.0 \mathrm{AU} / \mathrm{ml})$ PIVKA II values were prepared by mixing a plasma pool taken from patients undergoing warfarin treatment with normal plasma. These were then assayed in duplicate in each assay run. The interassay precision for these three pools was $9.8 \%$ for the low pool, $6.2 \%$ for the middle pool, and $9.0 \%$ for the high pool. With this assay, adult patients on stable anticoagulant treatment have PIVKA II values of $6-100 \mathrm{AU} / \mathrm{ml}$ with a mean of $40 \mathrm{AU} / \mathrm{ml}$.

Capillary whole blood prothrombin times (PTs) were measured during the home visits with a Biotrak coagulation monitor (Boehringer Mannheim, Indianapolis, Indiana, USA), which has been used and validated in adults and small children. ${ }^{15}{ }^{16}$ This monitor uses a disposable plastic reagent cartridge and one drop of whole blood. The PT is determined by the amount of time required for the blood to flow by capillary action through a channel between the initial reservoir and a mixing chamber that is coated with rabbit brain thromboplastin. The cessation of blood flow (clotting) is sensed by variation in the light scatter caused by movement of erythrocytes. The change in light scattering is detected by a photodetector and converted into an electrical 
Table 1 Mean (SD) vitamin $K_{1}$ plasma concentrations and INR values

\begin{tabular}{llll}
\hline & Oral & Intramuscular & pvalue $^{\star}$ \\
\hline Vitamin $\mathrm{K}_{1}(\mathrm{ng} / \mathrm{ml})$ & & & \\
$\quad 14$ days & $2.1(1.6)(\mathrm{n}=66)$ & $1.3(1.1)(\mathrm{n}=69)$ & $<0.007$ \\
30 days & $0.5(0.3)(\mathrm{n}=64)$ & $0.5(0.7)(\mathrm{n}=65)$ & $\mathrm{NS}$ \\
56 days & $0.5(0.8)(\mathrm{n}=63)$ & $0.2(0.2)(\mathrm{n}=66)$ & $<0.0003$ \\
INR & & & \\
14 days & $0.96(0.33)(\mathrm{n}=69)$ & $0.95(0.25)(\mathrm{n}=70)$ & $\mathrm{NS}$ \\
30 days & $0.86(0.18)(\mathrm{n}=66)$ & $0.84(0.14)(\mathrm{n}=71)$ & $\mathrm{NS}$ \\
56 days & $0.83(0.18)(\mathrm{n}=64)$ & $0.84(0.13)(\mathrm{n}=67)$ & $\mathrm{NS}$ \\
\hline
\end{tabular}

*Wilcoxon 2 sample test.

Table 2 Number of infants with positive PIVKA II $(\geqslant 0.1 \mathrm{AU} / \mathrm{ml})$

\begin{tabular}{lll}
\hline & Oral & Intramuscular \\
\hline Cord blood & $35(44 \%)$ & $40(52 \%)$ \\
14 days & 1 & 1 \\
30 days & 0 & 0 \\
56 days & 0 & 3 \\
\hline
\end{tabular}

One infant in the intramuscular group was positive at both 14 and 56 days. Cord blood values ranged from $<0.1$ to $66 \mathrm{AU} / \mathrm{ml}$.

signal that is analysed by a microprocessor and displayed on a screen as the PT and the international normalised ratio (INR). Infant haematocrits (autoanalyser, hospital haematology laboratory) were measured on the day of Biotrak measurements to ensure that the haematocrit was in the range recommended for the methodology $(\geqslant 23 \%$ and $\leqslant 53 \%)$. If Biotrak readings for PT were $<9$ seconds or $>14$ seconds (INR > 1.37), the PT was to be repeated immediately in the central coagulation laboratory after collection of a new sample by venepuncture. However, values for PT in our study never fell outside of this range on the Biotrak. To test the use of the Biotrak in newborn infants, 10 samples of cord blood were collected from 10 different elective caesarian section deliveries. One of these samples was discarded as the haematocrit was $>53 \%$. PT was measured simultaneously on the Biotrak and in the hospital coagulation laboratory. None of the paired samples had an INR difference of more than 0.33 INR units, the mean being 0.12 .

\section{STATISTICAL METHODS}

For statistical purposes, the following hypothesis was to be tested: oral Konakion MM is as effective as intramuscular Konakion in maintaining plasma vitamin $\mathrm{K}_{1}$ concentrations and plasma prothrombin times at 30 days. The Wilcoxon test was used, with a value of $\mathrm{p}<0.05$ being considered significant. We estimated that the probability of detecting plasma

Table 3 Subjects with positive PIVKA IIs $\geqslant 14$ days

\begin{tabular}{lllll}
\hline Subject & Birth & 14 days & 30 days & 56 days \\
\hline 102 (oral) & $\mathbf{1 0 . 8}$ & $\mathbf{0 . 1 1}$ & $<0.1$ & $<0.1$ \\
PIVKA (AU/ml) & ND & 0.75 & 0.32 & 0.10 \\
Vitamin K (ng/ml) & & & & \\
230 (IM) & $\mathbf{6 6 . 1}$ & $\mathbf{0 . 3 6}$ & $<0.1$ & $\mathbf{0 . 2}$ \\
PIVKA (AU/ml) & ND & 0.36 & 0.15 & 0.10 \\
Vitamin K (ng/ml) & $<0.1$ & $<0.1$ & $<0.1$ & $\mathbf{1 . 0 9}$ \\
19 (IM) & ND & 0.2 & 0.06 & $<0.05$ \\
PIVKA (AU/ml) & & $<0.1$ & $<0.1$ & $\mathbf{0 . 1}$ \\
Vitamin K (ng/ml) & $\mathbf{0 . 2 1}$ & 0.92 & 0.17 & 0.15 \\
$228(\mathrm{IM})$ & $\mathrm{ND}$ & 0.92 & \\
PIVKA (AU/ml) & & & & \\
Vitamin K (ng/ml) & & &
\end{tabular}

Positive values in bold.

$\mathrm{IM}$, intramuscular; ND, not done.
PIVKA II at 30 days after intramuscular administration of Konakion is small (as little as $5 / 10000$ neonates), so practical limitations did not allow us to use this difference as a study outcome, because more than a thousand infants would need to be enrolled. Because the analysis was an "intent to treat" one, dropouts were not replaced. Randomisation was continued until a minimum of 60 evaluable infants (completing the eight week study period) was obtained in each treatment group. Infants who completed at least two weeks of the study were included in the analysis.

\section{Results}

One hundred and fifty six infants (77 boys) were enrolled in the study, 79 in the oral group (mean (SD) birth weight, $3.6(0.6) \mathrm{kg}$ ) and 77 in the IM group (mean (SD) birth weight, 3.5 $(0.4) \mathrm{kg}$ ). Sixty seven infants in each group completed a full eight weeks of the study. Seven infants in the oral and one infant in the IM vitamin $\mathrm{K}$ groups dropped out before two weeks and could not be included in the data analysis. An additional 14 infants completed two weeks but less than eight weeks of the study, and were included in the analysis. All but two infants were white, there being one Hispanic infant in each group.

Table 1 shows the results of the vitamin $K_{1}$ plasma concentrations. The concentrations were highest on day 14 in both groups, although the concentrations in the oral group were significantly higher than the IM group. By day 30, there was no significant difference between the two groups, failing to disprove the null hypothesis. With an " $n$ " of 120 , the power to detect a difference of $0.30 \mathrm{ng} / \mathrm{ml}$ between the two groups was $85 \%$. Whereas the concentrations in the oral group had stabilised by 30 days, they continued to fall in the IM group until 56 days. At 56 days, the concentrations in the oral group were significantly higher than the IM group.

Table 2 shows the results of the PIVKA II analysis. Note the very high percentage of positive PIVKA II values at the time of birth-47\% of all infants. Eleven infants had cord blood values $>2.0 \mathrm{AU} / \mathrm{ml}$. This is in agreement with high detection rates in other studies. ${ }^{17}{ }^{18}$ There were only two positive PIVKA II values ( $\geqslant 0.1 \mathrm{AU} / \mathrm{ml}$ ) at 14 days (one in each group), none at 28 days, and three at 56 days (all in the IM group). Two of the positive values were in the same patient (patient 230) in the IM group (table 3 ). The limited number of subjects in this study precludes estimates of relative event rates of positive PIVKA II in these populations.

Table 3 shows the four subjects with raised PIVKA II values and their simultaneously measured vitamin $\mathrm{K}_{1}$ values. At 56 days, the three raised PIVKA II values were associated with low vitamin $\mathrm{K}_{1}(\leqslant 0.15 \mathrm{ng} / \mathrm{ml})$. However the raised PIVKA II values at 14 days (patients 102 and 230) were associated with normal concentrations of vitamin $K_{1}$ and raised concentrations of PIVKA II at birth.

Figure 1 shows the percentage of infants with low plasma vitamin $\mathrm{K}_{1}$ concentrations $(<0.15 \mathrm{ng} / \mathrm{ml})$ at 30 and 56 days of age. It 


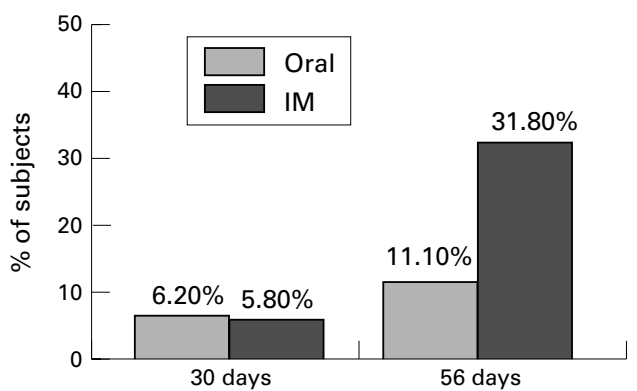

Figure 1 Percentage of infants with low plasma vitamin $K_{1}$ concentrations at 30 and 56 days.

should be noted that no infants had low vitamin $K_{1}$ values at 14 days. By 56 days, 21 of 28 of the low vitamin $K_{1}$ values were in the IM group, but only two of these infants had raised PIVKA II values (table 3). A total of seven infants in the oral group had low vitamin $\mathrm{K}_{1}$ concentrations, but there were no positive PIVKA II values in this group at 56 days.

Table 1 shows the INR data for the prothrombin times. Although there is a fall in both groups between days 14 and 30, the groups did not differ significantly at any time point. No infant had a haematocrit that was out of the range reported for use with the Biotrak coagulation monitor.

There were no major adverse events attributed to the vitamin $\mathrm{K}$ preparations used in this study, at least during the first 2 months of life. There were no bleeding episodes of any kind. Eight infants dropped out in the first two weeks of the study. Two of these developed intercurrent illnesses not related to the vitamin $\mathrm{K}$ administration. Of the remaining six infants, five were removed for protocol violations and one withdrew consent. An additional 14 infants dropped out after two weeks. One of these had an intercurrent illness, six were dropped for protocol violations, and seven mothers withdrew consent for their infants to continue in the study. Infants tolerated the oral medication very well. No infant "spat" the drug after administration, thus requiring removal from the study.

\section{Discussion}

The use of oral vitamin $\mathrm{K}_{1}$ for prophylaxis of the newborn infant has become more widespread following a report of an association between intramuscular vitamin $\mathrm{K}_{1}$ in the newborn and childhood cancer. ${ }^{19}$ However, other investigators in Europe and elsewhere have not confirmed the association, ${ }^{20-26}$ although a very small increased incidence of childhood leukaemia cannot be ruled out. ${ }^{27}{ }^{28}$ The American Academy of Pediatrics has recommended continued use of intramuscular prophylaxis, because there is no licensed product for oral prophylaxis in the USA. ${ }^{6}$ In Europe, the preparation studied in our report is now approved for use in some countries, although a preliminary report from Switzerland indicates several treatment failures in breast fed infants with cholestatic liver disease. ${ }^{29}$ In Switzerland, only two doses are given, both during the 1 st week of life, even though more than two doses are rec- ommended for breast feeding infants. ${ }^{30}$ Obviously, there is a need for an oral vitamin $\mathrm{K}$ product. This is the first study in infants to compare directly the new oral Konakion MM with a standard intramuscular preparation.

Our study has shown clearly that Konakion MM, when given three times in the first 30 days of life, maintains blood concentrations of vitamin $\mathrm{K}_{1}$ for the first 2 months of life at a concentration equal to or greater than that achieved with standard intramuscular prophylaxis. In fact, a third oral dose given at 30 days maintains the blood concentration of vitamin $K_{1}$ for 56 days, by which time the blood concentration in the intramuscular group has decreased significantly (table 1). This is important because late haemorrhagic disease after the administration of intramuscular preparations usually occurs during the first 3 months of life, and is often associated with intestinal malabsorption. ${ }^{1}$ We speculate that, when given in three doses, the mixed micellar form of vitamin $\mathrm{K}_{1}$ would prevent most cases of late haemorrhagic disease, especially as it has been used to maintain vitamin $\mathrm{K}$ status in older children with malabsorption secondary to cholestasis. ${ }^{10}$ However, in the normal newborn population, giving three doses of vitamin $\mathrm{K}_{1}$ to breast feeding infants, two of which would be given after hospital discharge in the USA, is problematic and a public health issue. Poor compliance in administering the three doses has already been reported. ${ }^{31} 32$

The most interesting findings in this study resulted from the PIVKA II analysis. We confirmed the high incidence of positive PIVKA II values in cord blood reported in other studies ${ }^{17}{ }^{18}$ (table 2). Eleven cord blood samples $(7.5 \%)$ had PIVKA II values that exceeded $2.0 \mathrm{AU} / \mathrm{ml}$, a value that is more than 20 times the "normal" background concentration. Three cord blood samples had PIVKA II values that exceeded $10.0 \mathrm{AU} / \mathrm{ml}$. However, why only half of the infants have low PIVKA II at birth, despite low vitamin $K_{1}$ plasma concentrations, is puzzling. The raised PIVKA II has been interpreted as a subclinical vitamin $\mathrm{K}$ deficiency in the newborn and goes along with the undetectable concentrations of vitamin $K_{1}$ in cord plasma with the assay used in this study. ${ }^{18}$ PIVKA II is the partially carboxylated form of the prothrombin molecule. Both vitamin $K_{1}$ and a liver enzyme, glutamyl carboxylase, are required for the formation of the active carboxylated form of prothrombin. ${ }^{1}$ Whether the very high rate of raised PIVKA II in cord blood represents a delay in maturation of the fetal liver enzyme glutamyl carboxylase, and/or is truly a result of inadequate transport of vitamin $\mathrm{K}_{1}$ across the placenta, remains a question for further study.

PIVKA values did not differ significantly between the two groups, although one could speculate on the meaning of three positive values in the IM group at day 56 compared with none in the oral group, especially as these three positive values were associated with low plasma vitamin $K_{1}$ concentrations (table 3 ). Unfortunately, our relatively small number of subjects does not allow us to predict accurately 
the incidence of positive PIVKA II in this population. This would require many thousands of infants in each group. However, three of 67 positive PIVKA II values at day 56 in the breast feeding IM group would represent a rate of positive PIVKA II values of 450/10 000 in this group at this time. These 450 infants would be at risk of haemorrhagic disease. This figure is very high compared with an incidence of late haemorrhagic disease of the newborn between $1 / 10000$ and $1 / 100000$ infants, dependent on vitamin $\mathrm{K}$ prophylaxis. ${ }^{7}$ There are no comparable data at 2 months of life in exclusively breast fed term infants using the PIVKA II assay. ${ }^{33}$

In this study of 156 infants, there were five positive PIVKA II values in four infants between 14 and 56 days of life (table 3). Infant 230, with a PIVKA II value of $66.1 \mathrm{AU} / \mathrm{ml}$ (confirmed with duplicate determinations), had the highest cord blood value ever measured in our laboratory. After an intramuscular injection, the value declined to $0.36 \mathrm{AU} / \mathrm{ml}$ at 14 days and was down to normal by day 30 $(<0.1 \mathrm{AU} / \mathrm{ml})$. However, by 56 days, the value in this infant had increased to $0.2 \mathrm{AU} / \mathrm{ml}$, reflecting the return of an abnormal vitamin $\mathrm{K}_{1}$ status. From the values in cord blood and on day 14 , it is possible to calculate that the half life of PIVKA II in this infant was 45.0 hours. This agrees with the value ( 46 hours) found in a previous study. ${ }^{18}$ A second infant (table 3) also had a very high PIVKA II value at birth $(10.8 \mathrm{AU} / \mathrm{ml})$. This infant was in the oral group and the PIVKA II value was still raised at 14 days $(0.11 \mathrm{AU} / \mathrm{ml})$. The calculated half life of PIVKA II in this infant was 50.8 hours. Thus, the two raised PIVKA II values at day 14 can be explained by the very high values at birth (>10 AU/ml). A third infant (data not shown) had a PIVKA II value of 15.0 in cord blood, which decreases to $<0.1 \mathrm{AU} / \mathrm{ml}$ by 14 days, the calculated half life again being about 45 hours.

As seen in table 3, raised PIVKA II values did not necessarily occur with low vitamin $\mathrm{K}_{1}$ values. In the two subjects with the highest cord blood concentrations of PIVKA II, the raised values at 14 days are associated with normal vitamin $K_{1}$ concentrations, reflecting the half life of PIVKA II of between 45 and 50 hours. On the other hand, the three raised PIVKA II values in the IM group at 56 days are all associated with low vitamin $\mathrm{K}_{1}$ plasma concentrations. In one of these patients (patient 19), the vitamin $K_{1}$ concentration is low at 30 days without PIVKA II being raised. This infant had an adequate vitamin $\mathrm{K}_{1}$ status at birth, which decreased to a subclinical deficiency state between 30 and 56 days, with a raised PIVKA II.

It appears that neither plasma vitamin $\mathrm{K}_{1}$ nor PIVKA II are ideal screening tools for subclinical vitamin $\mathrm{K}$ deficiency at birth. Many infants have "low" plasma vitamin $\mathrm{K}_{1}$ concentrations, particularly those who are exclusively breast fed after birth. ${ }^{34}$ All infants are born with low vitamin $\mathrm{K}_{1}$ plasma concentrations, and half of the infants in our study had detectable PIVKA II at this time. Thus, sensitivities are high and specificities are low for predicting haemorrhagic disease of the newborn. Furthermore, the relatively long half life of PIVKA II might also limit its usefulness as a screening test before 1 month of age, as discussed above. By 30 days of life, raised PIVKA II values are found in less than $10 \%$ of infants with low plasma vitamin $\mathrm{K}_{1}$ concentrations (fig 1). In adults, the correlation between PIVKA II and vitamin $\mathrm{K}_{1}$ concentrations has been reported to be weak $(r=-0.15$; $\mathrm{p}<0.05)$. $^{35}$

From our study, we can conclude the following about breast feeding infants receiving three oral vitamin $\mathrm{K}$ prophylaxis doses with Konakion MM: (1) plasma vitamin K concentrations were equal or significantly higher in orally supplemented babies compared with intramuscularly treated babies at the time points measured; and (2) up to 8 weeks of life, multiple doses of the new oral preparation maintain haemostasis and vitamin $\mathrm{K}$ status in breast fed infants at values that are equal to those achieved by the intramuscular preparation.

This study was funded by a grant from Hoffmann-La Roche Inc, Nutley, New Jersey, USA and Basel, Switzerland.

1 Greer FR. Vitamin K deficiency and hemorrhage in infancy. Clin Perinatol 1995;22:759-77.

2 Loughnan PM, McDougall PN. Epidemiology of late onset haemorrhagic disease: a pooled data analysis. $f$ Paediatr Child Health 1993;29:177-81.

3 Barton JS, McNinch AW, Tripp JH. Oral vitamin K prophylaxis and frequency of late vitamin $\mathrm{K}$ deficiency bleeding. Lancet 1994;343:1168.

4 Ekelund H. Late haemorrhagic disease in Sweden 19871989. Acta Paediatr Scand 1991;80:966-968.

5 Loughnan PM, McDougall PN. The efficacy of oral vitamin $\mathrm{K}_{1}$ : implications for future prophylaxis to prevent haemorrhagic disease of the newborn. F Paediatr Child Health 1993;29:171-6.

6 Vitamin K Ad Hoc Task Force. Controversies concerning vitamin $\mathrm{K}$ and the newborn. American Academy of Pediatrics. Pediatrics 1993;91:1001-3.

7 von Kries R, Göbel U. Repeated oral vitamin K prophylaxis in Germany. Acceptance and efficacy. In: Sutor AH, Hathaway W, eds. Vitamin $K$ in infancy. Stuttgart, Germany: FK Schattauer, 1995:197-206.

8 Hansen KN, Ebbesen F. Neonatal vitamin K prophylaxis in Denmark: three years' experience with oral administration Denmark: three years' experience with oral administration
during the first three months of life compared with one oral administration at birth. Acta Paediatr 1996;85:1137-9.

9 McNinch AW, Upton C, Samuels M, et al. Plasma concenMcNinch AW, Upton C, Samuels $M$, et al. Plasma concen-
trations after oral or intramuscular vitamin $\mathrm{K}_{1}$ in neonates. trations after oral or intramuscular
Arch Dis Child 1985;60:814-18.

10 Amédée-Manesme O, Lambert WE, Alagille D, et al. Pharmacokinetics and safety of a new solution of vitamin $\mathrm{K}_{1}$ in children with cholestasis. F Pediatr Gastroenterol Nutr 1992; 14:160-5.

11 Schubiger G, Tönz O, Grüter J, et al. Vitamin $\mathrm{K}_{1}$ concentration in breast-fed neonates after oral or intramuscular administration of a single dose of a new mixed-micellar preparation of phylloquinone. F Pediatr Gastroenterol Nutr 1993;16:435-9.

12 McCarthy PT, Harrington DJ, Shearer MJ. Assay of phylloquinone in plasma by HPLC with electrochemical quinone in plasma by Hection. In: McCormick DB, Suttie JW, Wagner C, eds. detection. In: McCormick DB, Suttie JW,
Methods in enzymology 1997;282:421-35.

13 Shearer MJ. Phylloquinone (vitamin $K_{1}$ ) in serum or plasma by HPLC. In: Fidanza F, ed. Nutritional status assessment - a manual for population studies. London: Chapman \& Hall, manual for populd

14 Motahara K, Kuroki Y, Kan H, Endo F, Matsuda I. Detection of vitamin $\mathrm{K}$ deficiency by use of an enzyme-linked immunosorbent assay for circulating abnormal prothrombin. Pediatr Res 1985;19:353-7.

15 McCurdy SA, White RH. Accuracy and precision of a portable anticoagulation monitor in a clinical setting. Arch Intern Med 1992;152:589-92.

16 Massicotte P, Marzinotto V, Vegh P, Adams M, Andrew M. Home monitoring of warfarin therapy in children with a whole blood prothrombin monitor. F Pediatr 1995;127: 389-94.

17 Belle M, Leclercq M, Vignal B, et al. Application of an ELISA test to Vitamin $\mathrm{K}$ deficient conditions using a new monoclonal antibody against human des-gamma-carboxyprothrombin (DCP). In: Sutor AH, Hathaway W, eds. prothrombin (DCP). In: Sutor AH, Hathaway W, eds.
Vitamin $K$ in infancy. Stuttgart, Germany: FK Schattauer, 1995:123-38. 
18 Cox A, McCarthy PT, Harrington DJ, Shearer MJ. Human vitamin $\mathrm{K}$ status assessment: value of plasma des-gamma-
carboxyprothrombin and vitamin $\mathrm{K}_{1}$. Int $\mathcal{F}$ Vitam Nutr Res carboxyprothrom

19 Golding J, Greenwood R, Birmingham K, et al. Childhood cancer, intramuscular vitamin $\mathrm{K}$, and pethidine given during labour. BMF 1992;305:341-6.

20 Ekelund H, Finnström O, Gunnarskog J, et al. Administration of vitamin $\mathrm{K}$ to newborn infants and childhood cancer. BMF 1993;307:89-91.

21 Klebanoff MA, Read JS, Mills JL, et al. The risk of childhood cancer after neonatal exposure to vitamin K. N Engl f Med 1993;329:905-8.

22 Olsen JH, Hertaz H, Blinkenberg K, Verder H. Vitamin K regimens and incidence of childhood cancer in Denmark. BMF 1994;308:895.

23 Von Kreis R, Göbel U, Hachmeister A, Kaletsh U, Michaelis J. Vitamin K and childhood cancer: a population based case-control study in Lower Saxony, Germany. BMF 1996; 313:199-203

24 Ansell P, Bull D, Roman E. Childhood leukemia and intramuscular vitamin $\mathrm{K}$ : findings from a case control study. BMF 1996;313:204-5.

25 McKinney PA, Juszczak E, Findlay E, Smith K. Casecontrol study of childhood leukaemia and cancer in Scotland: findings for neonatal intramuscular vitamin $\mathrm{K}$. BMF 1998;316:173-7.

26 Passmore SJ, Draper G, Brownbill P, Kroll M. Ecologica studies of relation between hospital policies on neonatal vitamin $\mathrm{K}$ administration and subsequent occurrence of childhood cancer. BMF 1998;316:184-9.
27 Passmore SJ, Draper G, Brownbill P, Kroll M. Case-control studies of relation between childhood cancer and neonatal vitamin K administration. BMf 1998;316:178-84.

28 Parker L, Cole M, Craft W, Hey EN. Neonatal vitamin K administration and childhood cancer in the north of England: retrospective case-control study. BMF 1998;316: 189-93.

29 Baenziger O, Braegger CP, Fanconi S. Oral vitamin K prophylaxis for newborn infants: safe enough? Lancet 1996; 348:1456.

30 Schubiger G, Tönz O, Grüter J, Shearer MJ. Vitamin K and PIVKA II concentrations in breast-fed infants after prophylaxis with different preparations of vitamin $\mathrm{K}$. In: Sutor $\mathrm{AH}$, Hathaway W, eds. Vitamin $K$ in infancy. Stuttgart, Germany: FK Schattauer, 1995:339-45.

31 Croucher C, Azzopardi D. Compliance with recommendations for giving vitamin $\mathrm{K}$ to newborn infants. BMF 1994; 308:894-5.

32 Hill RJ. The uptake of the third oral vitamin $\mathrm{K}$ dose in general practice. NZ Med F 1994;107:177-8.

33 Von Kries R, Greer FR, Suttie JW. Assessment of vitamin K status of the newborn infant. F Pediatr Gastroenterol Nutr 1993;16:231-8.

34 Greer, FR, Marshall S, Suttie JW. Improving the vitamin K status of breast-feeding infants with maternal vitamin $\mathrm{K}$ supplements. Pediatrics 1997;99:88-92.

35 Sokol LJ, Sadowski JA. Comparison of biochemical indexes for assessing vitamin $\mathrm{K}$ nutritional status in a healthy adult population. Am f Clin Nutr 1996;63:566-73. 